\title{
Prenatal diagnosis of a de novo trisomy 20p detected by noninvasive prenatal testing
}

\author{
xu yan ${ }^{1}$, Haiying Peng ${ }^{2}$, and Changjun Zhang ${ }^{2}$ \\ ${ }^{1}$ Remin Hospital, Hubei University of MedicineShiyan \\ ${ }^{2}$ Renmin Hospital, Hubei University of Medicine
}

July 16, 2020

\begin{abstract}
Trisomy 20p, resulting from duplication of all or part of the short arm of chromosome 20, is a rare chromosomal disorder while there is little case of trisomy 20p in prenatal diagnosis. Here we report firstly a case of de novo trisomy $20 \mathrm{p}$ detected by noninvasive prenatal testing.
\end{abstract}

Key Clinical Message

Prenatal diagnosis of trisomy $20 \mathrm{p}$ seems to be difficult, considering the capacity of ultrasound to detect mild dysmorphy. NIPT has good performance in detecting fetal trisomy $20 \mathrm{p}$ combined with low coverage WGS and karyotype analysis.

\section{Introduction}

Trisomy 20p manifesting as intellectual disability and speech delay is a rare genetic disorder ${ }^{1,2}$. The cases of trisomy 20p previously reported mostly focused on the patients after birth and there is little information about prenatal screening of trisomy 20p considering the lack of effective detection technology.

Since 2011, NIPT has been introduced into clinical practice for the detection of fetal trisomy 21, 18, and 13 via massively parallel sequencing of cell-free DNA (cfDNA) in maternal plasma ${ }^{3}$. The fetal CNVs can also be detected due to the statistical power of the methods published and bigger sequencing depth ${ }^{4}$. Using two to eight million reads, Chen et al. detected fetal CNVs bigger than $10 \mathrm{Mb}^{5}$. The trisomy $20 \mathrm{p}$ can be screened theoretically considering the short arm of chromosome 20 is bigger than $10 \mathrm{Mb}$ and the mean sequencing reads of NIPT is bigger than 3.5 million reads.

Here we firstly report a case of de novo trisomy 20p detected by NIPT in a 27 years pregnant women, confirmed by prenatal diagnosis.

\section{Clinical report}

A 27-year-old pregnant woman (G1P0) with gestational age (GA) of 16 weeks 2 days accepted NIPT in reproductive medicine center of renmin hospital, hubei university of medicine. The couple with no special face and normal intelligence is not consanguineous and both healthy. The fetal nuchal translucency (NT) thickness is $1.2 \mathrm{~mm}$ with GA of 13 weeks. 
$5 \mathrm{ml}$ blood was collected in cfDNA storage tube (CW2814M) from the pregnant woman. The cfDNA was extracted from plasma, followed by end repair, adaptors ligation and PCR amplification according to the instruction. Single-ended sequencing was performed on BGISEQ-500 (BGI-Wuhan, Wuhan, China) with the $35 \mathrm{bp}$ length. The raw sequence reads is 11.91 million, of which $74.2 \%$ is blasted to the human genome. The effective reads is 7.98 million and the fetal fraction in the maternal plasma is $9.35 \%$. The Z-score of T21, T18 and T13 were less than 3 which suggested 21-trisomy, 18-trisomy and 13-trisomy were negative while the $\mathrm{Z}$ value of T20 is 5.508 which meaned that there was about a dupication of chromosome 20 (Figure 1A).

Following genetic counselling, the amniocentesis was performed at 17 weeks of gestation under the guidance of ultrasound. STR analysis via Multiple STR genotyping kit (DARUI biotechnology) suggested there was no maternal cell contamination in amniotic fluid which was used to cell culture analysis of fetal amniotic fluid karyotype and CNVs analysis by LC-WGS ${ }^{6-8}$.

Cytogenetic analysis by G-banding shows the karyotype of the fetus with 46, XX, add(21)(p12) (Figure 1B). The karyotype analysis of the couple showed no obvious abnormalities.

CNVs sequencing was performed on a BGISeq-500 platform (BGI-Wuhan, Wuhan, China) as previously reported. In brief, $1 \mu \mathrm{g}$ genomic DNA extracted from fetal amniotic fluid cell was randomly fragmented. Library were conducted according to the standard instruction and sequenced with a minimum of $\sim 15$ million reads (single-end $50 \mathrm{bp}$ ) per sample ${ }^{8}$. The homozygous/heterozygous duplication and deletion was detected by reported method6. The CNV-Seq analysis results was seq[GRCh37/hg19]20p11.22p13 $(60,001-21,474,604) \times 3$, indicating a 21.4 Mb duplication on chromosome 20p11.22-p13 (Figure 1C).

At 22 weeks of gestation, an isolated choroid plexus cyst measuring $0.41 \mathrm{~cm} \times 0.36 \mathrm{~cm}$ in fetal brain was identified during routine sonography.

\section{Discussion}

Since the 1980s, chromosomal microdeletions/microduplications have been be identified to associated with developmental delay and intellectual disability ${ }^{9}$. The common use of next-generation sequencing (NGS) and array comparative genomic hybridization $(\mathrm{aCGH})$ in diagnostics has led to an increase of known pathogenic $\mathrm{CNVs}^{10}$. Thanks to the application of NIPT, fetal chromosomal aneuploidies subchromosomal abnormalities can be screening with high sensitivity and specificity ${ }^{11,12}$.

Trisomy 20p is a rare chromosomal duplication manifesting as poor coordination, mental retardation with speech delay, and moderate facial dysmorphisms which emerged from post-natal observations ${ }^{13,14}$. There is only one prenatal case of trisomy $20 \mathrm{p}$ resulting from maternal inversion [46,XX,inv $(20)(\mathrm{p} 11.2 \mathrm{q} 13.3)]$ in chromosome 20 . The only ultrasonographic anomaly was an increased NT of $3.6 \mathrm{~mm}$ at 12 weeks ${ }^{15}$.

Here we report firstly a case of prenatal diagnosis of de novo trisomy 20p result from the parents with normal karyotype. The fetal karyotype was $46, \mathrm{XX}$, add(21)(p12), indicating additional unknown origin was attached to band 21p12. The CNV-Seq result revealed the additional unknown origin may be 20p11.22-p13. The only ultrasonographic anomaly at 21 weeks was an choroid plexus cyst located on the left side of the brain while the NT value is normal. The fetus after induction had normal phenotypes. According to postnatal phenotypes described, prenatal diagnosis of such partial trisomy 20p seems to be difficult, considering the capacity of ultrasound to detect mild dysmorphy. However, NIPT has good performance in detecting fetal trisomy 20p combined with LC-WGS of amniotic fluid cell.

Further via deeper sequencing fetal subchromosomal CNVs can be detected by NIPT for the lower sequencing cost. NIPT, combined with karyotype analysis and NGS may be a efficient screening strategy for fetal trisomy 20 p syndrome.

Acknowledgements: We thank the family for their participation and for allowing us to report their family's data.

\section{Author Contributions}


Conception and design: XY, CJZ

Analysis and interpretation: XY, HYP

Data collection: HYP

Writing the article: XY

Critical revision of the article: CJZ

Final approval of the article: CJZ

Statistical analysis: XY

Conflict of interest: No conflicts of interest

Funding: The Talent Research Starting Foundation (2016QDJZR09), Hubei University of Medicine (2016).

\section{References:}

1. Bartolini L, Sartori S, Lenzini E, et al. De novo trisomy 20p characterized by array comparative genomic hybridization: report of a novel case and review of the literature. Gene. 2013 Jul 25;524(2):368-72.

2. Choi J, Yoon SY, Park BG, et al. De Novo Pure Trisomy 20p: Report of a Novel Case of a Marker Chromosome and Literature Review. Ann Lab Med. 2020 May;40(3):277-280.

3. Lo YM. Non-invasive prenatal testing using massively parallel sequencing of maternal plasma DNA: from molecular karyotyping to fetal whole-genome sequencing. Reprod Biomed Online. 2013 Dec;27(6):593-8.

4. Li R, Wan J, Zhang Y, et al. Detection of fetal copy number variants by non-invasive prenatal testing for common aneuploidies. Ultrasound Obstet Gynecol. 2016 Jan;47(1):53-7.

5. Lo KK, Karampetsou E, Boustred C, et al. Limited Clinical Utility of Non-invasive Prenatal Testing for Subchromosomal Abnormalities. Am J Hum Genet. 2016 Jan 7;98(1):34-44.

6. Donaghue C, Roberts A, Mann K, et al. Development and targeted application of a rapid QF-PCR test for sex chromosome imbalance. Prenat Diagn. 2003 Mar;23(3):201-10.

7. Zhao G, Dai P, Gao S, et al. A case of prenatal diagnosis of $18 p$ deletion syndrome following noninvasive prenatal testing. Mol Cytogenet. 2019;12:53.

8. Wang H, Dong Z, Zhang R, et al. Low-pass genome sequencing versus chromosomal microarray analysis: implementation in prenatal diagnosis. Genet Med. 2020 Mar;22(3):500-510.

9. Watson CT, Marques-Bonet T, Sharp AJ, et al. The genetics of microdeletion and microduplication syndromes: an update. Annu Rev Genomics Hum Genet. 2014;15:215-244.

10. Weise A, Mrasek K, Klein E, et al. Microdeletion and microduplication syndromes. J Histochem Cytochem. 2012 May;60(5):346-58.

11. Yin AH, Peng CF, Zhao X, et al. Noninvasive detection of fetal subchromosomal abnormalities by semiconductor sequencing of maternal plasma DNA. Proc Natl Acad Sci U S A. 2015 Nov 24;112(47):146705 .

12. Yu D, Zhang K, Han M, et al. Noninvasive prenatal testing for fetal subchromosomal copy number variations and chromosomal aneuploidy by low-pass whole-genome sequencing. Mol Genet Genomic Med. 2019 Jun;7(6):e674.

13. Oppenheimer S, Dignan P, Soukup S. Partial trisomy 20p: familial occurrence. Am J Med Genet. 2000 Dec 11;95(4):316-9.

14. Chaabouni M, Turleau C, Karboul L, et al. De novo trisomy 20p of paternal origin. Am J Med Genet A. 2007 May 15;143A(10):1100-3.

15. Molina-Gomes D, Nebout V, Daikha-Dahmane F, et al. Partial trisomy 20p resulting from recombination of a maternal pericentric inversion: case report of a prenatal diagnosis by chorionic villus sampling. Prenat Diagn. 2006 Mar;26(3):239-41.

Figure 1 . The prenatal screening and prenatal diagnosis results of patient(A-The T-score of chromosome 20 detected by NIPT; B-Karyotype analysis of maternal amniotic fluid (46, XX, add(21)(p12)); C-Copy number 
variation of maternal amniotic fluid showing that a duplication of $21.41 \mathrm{Mb}$ on chromosome 20p11.22-p13)

A

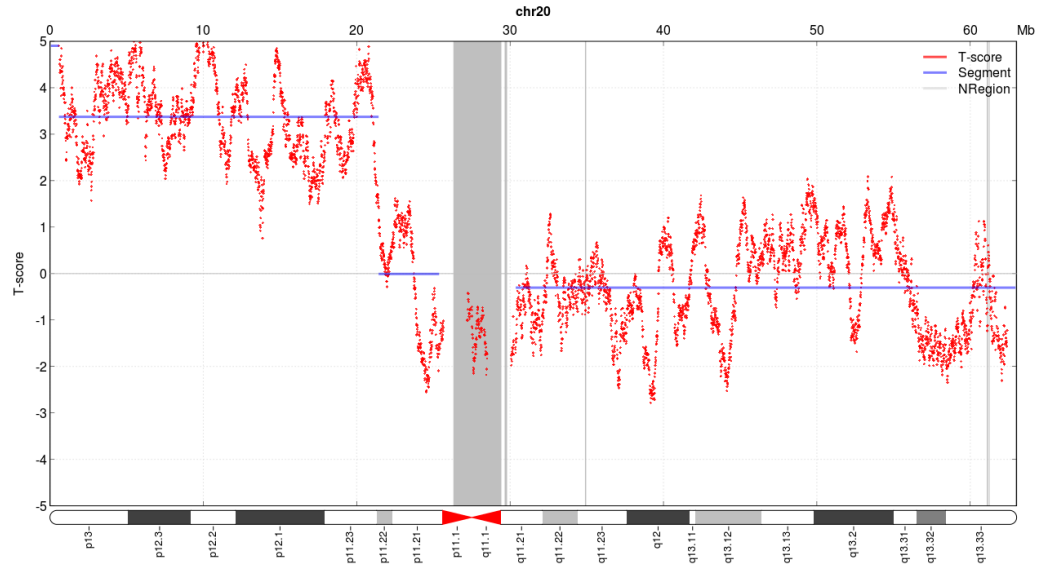

B

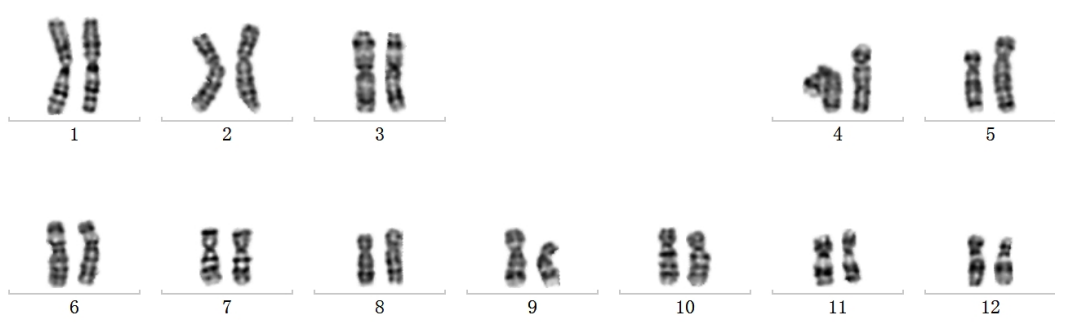

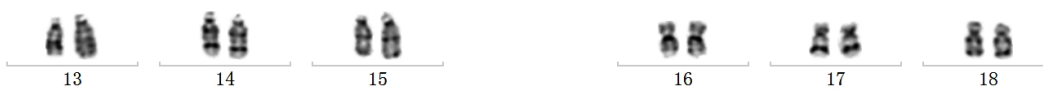

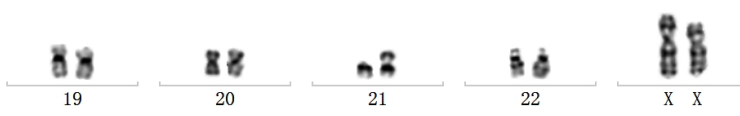

C

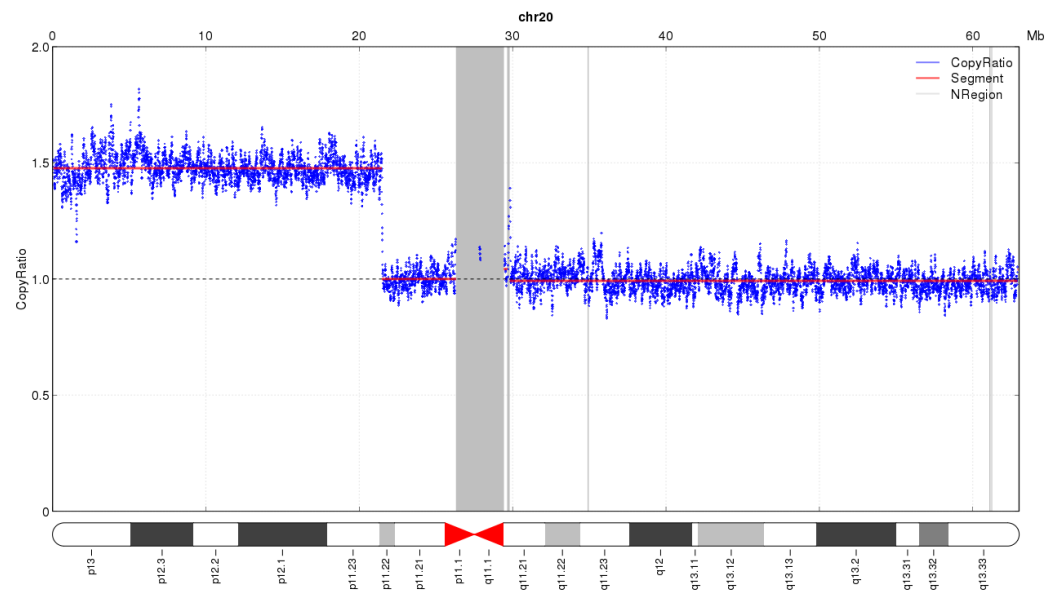

